\title{
Efficacy of Venetoclax Combined with Decitabine-Based Treatment for Heavily Pre-Treated Relapsed or Refractory AML Patients in a Real-World Setting
}

\author{
Juan Tong $(\mathbb{D}$ \\ $\mathrm{Na}$ Zhao \\ Xing $\mathrm{Hu}$ \\ Wen Yao \\ Yaxin Cheng \\ Li Zhou (D) \\ Huilan Liu \\ Liangquan Geng \\ Zimin Sun \\ Changcheng Zheng
}

Department of Hematology, The First Affiliated Hospital of USTC, Division of Life Sciences and Medicine, University of Science and Technology of China, Hefei, People's Republic of China
Correspondence: Changcheng Zheng Department of Hematology, The First Affiliated Hospital of USTC, Division of Life Sciences and Medicine, University of Science and Technology of China, Lujiang Road No. 17, Hefei, 23000I, People's Republic of China

Tel +86-55I-62284476

Fax +86-55I-62284476

Email zhengchchII23@ustc.edu.cn
Purpose: We report the efficacy and safety of venetoclax plus decitabine-based treatment in heavily pre-treated relapsed or refractory acute myeloid leukaemia (RR-AML) in a realworld setting.

Patients and Methods: There were 22 patients in this study and the median age was 47.5 (12-84) years old, including 11 males and 11 females. Among them, 8 patients were relapsed AML including 2 patients relapsed after HSCT and 14 patients with primary refractory AML including 4 secondary AML. The median number of cycles of previous chemotherapy was 4 (range, 2-10).

Results: After a course of venetoclax plus decitabine-based treatment, 9 patients achieved complete remission (CR) and 1 patient achieved complete remission with incomplete haematological recovery (CRi). The overall response rate (ORR) was $45.5 \%$ and the $\mathrm{CR}$ rate was $40.9 \%$, and the median time to reach CR/CRi was $21(13-46)$ days. Four of the $10 \mathrm{CR} /$ CRi patients relapsed again, and the median time of relapse was $5(1.0-24)$ months. The oneyear overall survival rate was $31.8 \%$, and the median survival time was 6 months $(95 \% \mathrm{CI}$, 1-9 months). The one-year overall survival rate of $10 \mathrm{CR} / \mathrm{CRi}$ patients was $59.1 \%$, and the 12 NR patients was $10.4 \%(\mathrm{p}=0.001)$. Nausea and vomiting occurred in 11 patients $(50.0 \%)$. All patients had grade IV neutropenia and IV thrombocytopenia (100\%). Pneumonia occurred in 14 patients $(63.6 \%)$ and septicaemia occurred in 2 patients $(9.0 \%)$. The cause of death in all patients was primary disease progression, and no patients died due to the side effects.

Conclusion: The efficacy of venetoclax plus decitabine-based treatment in the real-world treatment of heavily pre-treated RR-AML is similar to that in clinical trials, and the side effects are controllable.

Keywords: venetoclax, hypomethylating agents, acute myeloid leukaemia

\section{Introduction}

Acute myeloid leukaemia (AML) accounts for approximately $80 \%$ of acute leukaemias in adults and is heterogeneous. Generally, the complete remission rate (CR) of 1-2 courses of standard-dose induction chemotherapy can reach $60-70 \%$, but approximately $30-40 \%$ of patients cannot achieve CR with conventional chemotherapy. ${ }^{1}$ AML patients who cannot achieve CR with two courses of the standard regimen are classified as primary refractory AML. In addition, regardless 
of whether high-dose chemotherapy or haematopoietic stem cell transplantation (HSCT) is used for consolidation treatment, $20-30 \%$ of patients will still have disease relapse, although $30-40 \%$ of patients with relapse can achieve remission again through a second-line regimen. The second-line regimen in relapsed AML mainly includes a high-dose cytarabine-based regimen, such as a fludarabine/cladribine+ cytarabine + granulocyte colonystimulating factor (FLAG/CLAG) regimen, and a priming regimen, but most patients do not achieve $\mathrm{CR}$ with this regimen. ${ }^{2}$ At present, the treatment of refractory and relapsed AML (RR-AML) is difficult. In recent years, a number of clinical trials have shown that the specific Bcl-2 inhibitor venetoclax combined with hypomethylating agents (HMA) is effective in the treatment of relapsed and primary refractory AML. However, there is obvious heterogeneity in the reports of treatment efficiency, the overall response rate (ORR) ranges from $21 \%$ to $75 \%{ }^{3,4}$ In this study, we published the data on the efficacy of venetoclax combined with a hypomethylating agent decitabine in heavily pre-treated patients with RR-AML in a real-world setting; and we also performed a systematic literature review to assess the overall response rates (ORR) and the impact of genes mutations on RR-AML patients treated with venetoclax + HMA.

\section{Methods}

\section{Patients}

From May 2018 to January 2021, twenty-two patients with heavily pre-treated relapsed or refractory AML who received venetoclax plus decitabine-based treatment were collected. These patients had one of the following clinical characteristics: Relapsed AML patients failed to achieve CR after one or more courses of the second-line treatment after the first relapse; AML patients relapsed after HSCT; newly diagnosed AML patients who failed to reach CR after two courses or more of standard induction chemotherapy (primary refractory disease). ${ }^{5}$ Demographic characteristics, blood cell counts, proportions of blast cells in the bone marrow, initial induction chemotherapy regimens, median remission times, time to relapse durations, reinduction therapy and chemotherapy schemes after relapse, and total chemotherapy courses were collected. All treatment options were agreed upon and signed by patients and their families. The research was approved by the ethics committee of the first affiliated hospital of university of science and technology of China and all patients had given written informed consent. Among them, children under 18 years old had obtained the informed consent from their parents or legal guardians. This study was conducted in accordance with the Declaration of Helsinki.

\section{Treatment}

Treatment regimens included venetoclax (100 $\mathrm{mg}$ on day 1 and $200 \mathrm{mg}$ on day 2, and $400 \mathrm{mg}$ scheduled for days 3 to 28) and decitabine (at a dose of $15 \sim 20 \mathrm{mg} / \mathrm{m} 2$ per day on days 1 through 5 every 28-day cycle).

On the basis of venetoclax plus decitabine, three patients younger than 50 years with good physical fitness (ECOG score $0-1)$ received cladribine $\left(5 \mathrm{mg} / \mathrm{m}^{2}\right.$ per day on days 1 through 5) and low-dose cytarabine $\left(10 \mathrm{mg} / \mathrm{m}^{2} \mathrm{q} 12 \mathrm{~h}\right.$ on days 1 through 10 or 14); Six patients were treated with the FLT3 inhibitor sorafenib (400mg scheduled for days 1 to 28) due to the FLT3-ITD mutations.

To prevent the occurrence of tumour lysis syndrome, venetoclax treatment was started when the white blood cell count was less than $25 \times 10^{9} / \mathrm{L}$ and hydration alkalization and diuresis were also administered during the treatment. Posaconazole or voriconazole was given to prevent fungal infection. When there was severe neutropenia or the patient develops severe infections, venetoclax was temporarily stopped.

\section{Follow-Up and Statistical Analysis}

The patients were followed up to April 10, 2021. The median follow-up time was 4 (1-36) months after venetoclax combined with decitabine treatment, and no patient was lost to follow up. After treatment, routine blood tests were conducted every 1-2 days; liver and kidney function and electrolyte and blood glucose levels were detected 2-3 times a week; and bone marrow cytology and minimal residual disease (MRD) were detected every month after treatment. The main followup measures were ORR [including CR, CRi, and partial remission (PR)], survival time and adverse events. We used Common Terminology Criteria for Adverse Events (CTCAEVersion 5.0) to grade and evaluate the adverse events (AE). SPSS 22.0 software was used for statistical analysis, and the Kaplan-Meier method was used to generate survival curves. The overall survival (OS) time was defined as the time from the beginning of treatment to the end of follow-up or death.

\section{Results}

\section{Characteristics of Patients}

There were 11 males and 11 females with a median age of 47.5 years (12-84 years). Among them, 8 patients were 
relapsed AML including 2 patients relapsed after HSCT and 14 patients were primary refractory AML including 4 secondary AML. The median number of cycles of previous chemotherapy was 4 (range, 2-10). The median percentage of blast cells in the bone marrow was 56 (2898) \%. Gene mutations included FLT3-ITD mutation in 6 cases, FLT3-TKD mutation in 1 case, NPM1 mutation in 7 cases, IDH1/IDH2 mutation in 3 cases, AML-ETO mutation in 2 case, DNMT3A mutation in 2 cases, TP53 mutation in 1 case, KRAS mutation in 1 case, MLL-AF6 mutation in 1 case, TET-2 mutation in 1 case, SRSF2 mutation in 1 case, CBF $\beta$-MYH11 mutation in 1 case, CEBPA mutation in 1 case, and ASXL1 mutation in 1 case. (Tables 1 and 2).

Before treatment with decitabine and a Bcl-2 inhibitor, 8 patients presented with hyperleukocytosis, and 5 of these patients had leukocytes $>100 \times 10^{9} / \mathrm{L}$. They were treated with cytarabine $(100 \mathrm{mg})$ and etoposide $(100 \mathrm{mg})$ to reduce the white blood cell counts to less than $25 \times 10^{9} / \mathrm{L}$ before venetoclax treatment.

\section{Remission Rates and Survival Time}

After a course of the treatment, 9 patients achieved complete remission (CR) and 1 patient achieved complete remission with incomplete haematological recovery (CRi). The overall response rate (ORR) was $45.5 \%$ and the $\mathrm{CR}$ rate was $40.9 \%$, and the median time to reach $\mathrm{CR} /$ CRi was 21 (13-46) days. Seven patients achieved CR at 28th day after venetoclax combined with decitabine treatment and the CR rate at 28th day was $31.8 \%$. All CR patients achieved complete molecular remission or a minimal residual disease (MRD)-negative status. Three patients successfully received HSCT after CR, and the others continued venetoclax plus decitabine maintenance treatment.

The one-year overall survival rate was $31.8 \%$, and the median survival time was 6 months ( $95 \%$ CI, $1-9$ months) (Figure 1). Four of the $10 \mathrm{CR} / \mathrm{CRi}$ patients relapsed again, and the median time of relapse was 5 (1.0-24) months. The one-year overall survival rate of $10 \mathrm{CR} / \mathrm{CRi}$ patients

Table I The Characteristics of All the Patients and the Comparison of the CR/CRi and the NR Patients

\begin{tabular}{|c|c|c|c|c|}
\hline Characteristics & $\mathbf{N}=\mathbf{2 2}$ & CR/CRi(N=10) & $N R(N=12)$ & $P$ value \\
\hline Age(years) & $47.5(12-84)$ & $48(|2-8|)$ & $47(24-84)$ & I \\
\hline Male, n (\%) & II (50\%) & $4(40.0 \%)$ & $7(58.3 \%)$ & 0.67 \\
\hline Numbers of chemotherapy in the past & $4(2-10)$ & $3(2-10)$ & $7(3-7)$ & 0.05 \\
\hline AML type & & & & 0.09 \\
\hline Primary AML & $18(81.8 \%)$ & $10(100 \%)$ & $8(66.7 \%)$ & \\
\hline Secondary AML & $4(18.2 \%)$ & $0(0 \%)$ & $4(33.3 \%)$ & \\
\hline Relapse or refractory AML & & & & 0.44 \\
\hline Relapse AML & $8(66.7 \%)$ & $5(50.0 \%)$ & $3(25.0 \%)$ & \\
\hline Refractory AML & $14(63.6)$ & $5(50.0 \%)$ & $9(75.0 \%)$ & \\
\hline Blast cells in the BM before treatment (\%) & $56(28-98)$ & $60(29-98)$ & $56(28-98)$ & 0.49 \\
\hline Prior HMAs, n (\%) & I7(77.3\%) & $6(60.0 \%)$ & $\mathrm{II}(9 \mathrm{I} .7 \%)$ & 0.21 \\
\hline WBC count at onset & $\mid 3.8(|.38-| 7 \mid)$ & $40.63(2.3-170)$ & $4.27(|.38-| 7 \mid)$ & 0.10 \\
\hline ELN risk, n (\%) & & & & 0.64 \\
\hline Favorable & $5(22.7 \%)$ & $3(30.0 \%)$ & $2(16.7 \%)$ & \\
\hline Intermediate & $7(31.8 \%)$ & $2(20.0 \%)$ & $5(41.7 \%)$ & \\
\hline Adverse & $10(45.5 \%)$ & $5(50.0 \%)$ & $5(41.7 \%)$ & \\
\hline FLT3 mutant, n (\%) & $7(31.8 \%)$ & $5(50.0 \%)$ & $2(16.7 \%)$ & 0.17 \\
\hline NPMI mutant, n (\%) & $7(31.8 \%)$ & $5(50.0 \%)$ & $2(16.7 \%)$ & 0.17 \\
\hline
\end{tabular}




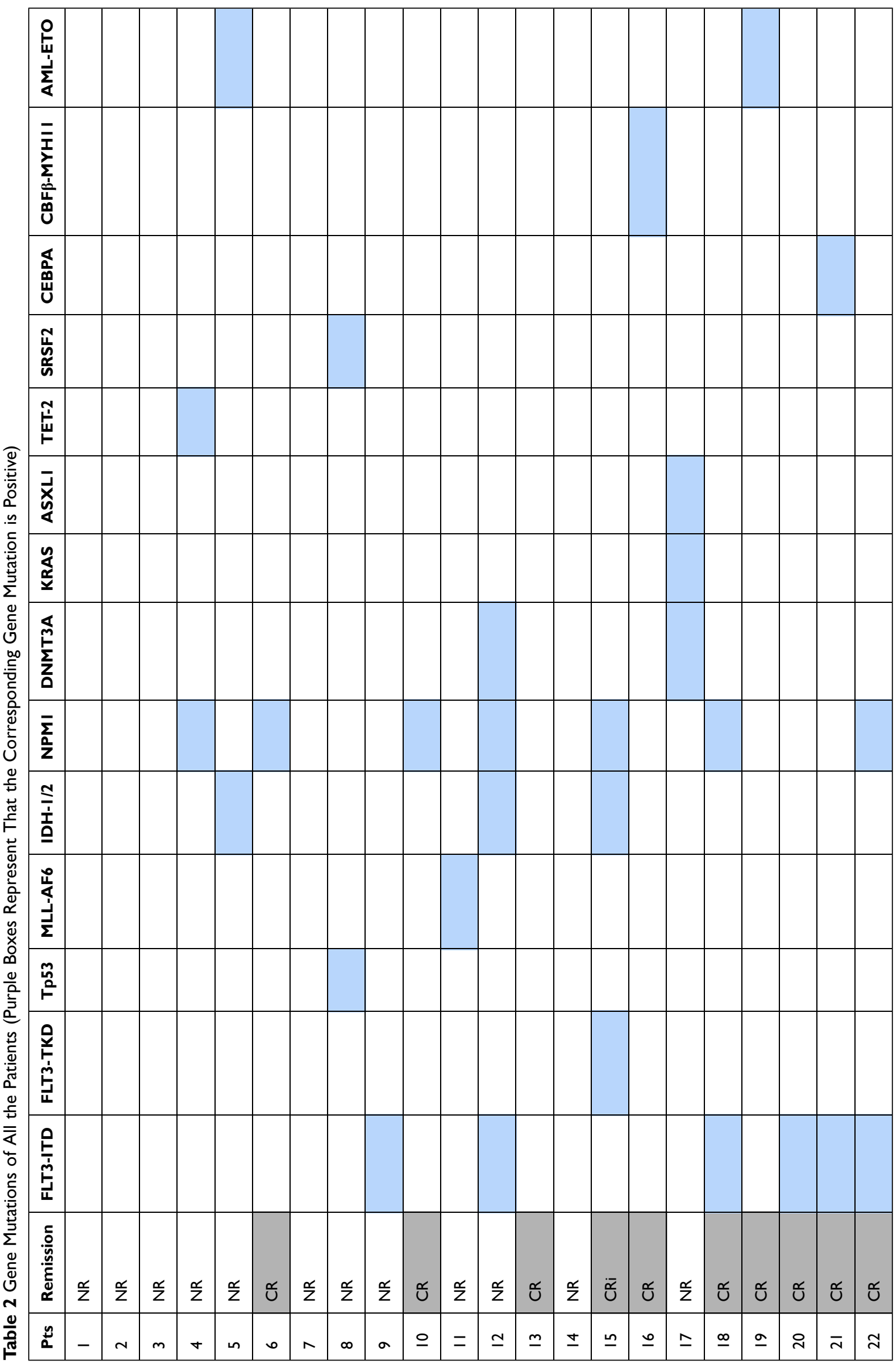




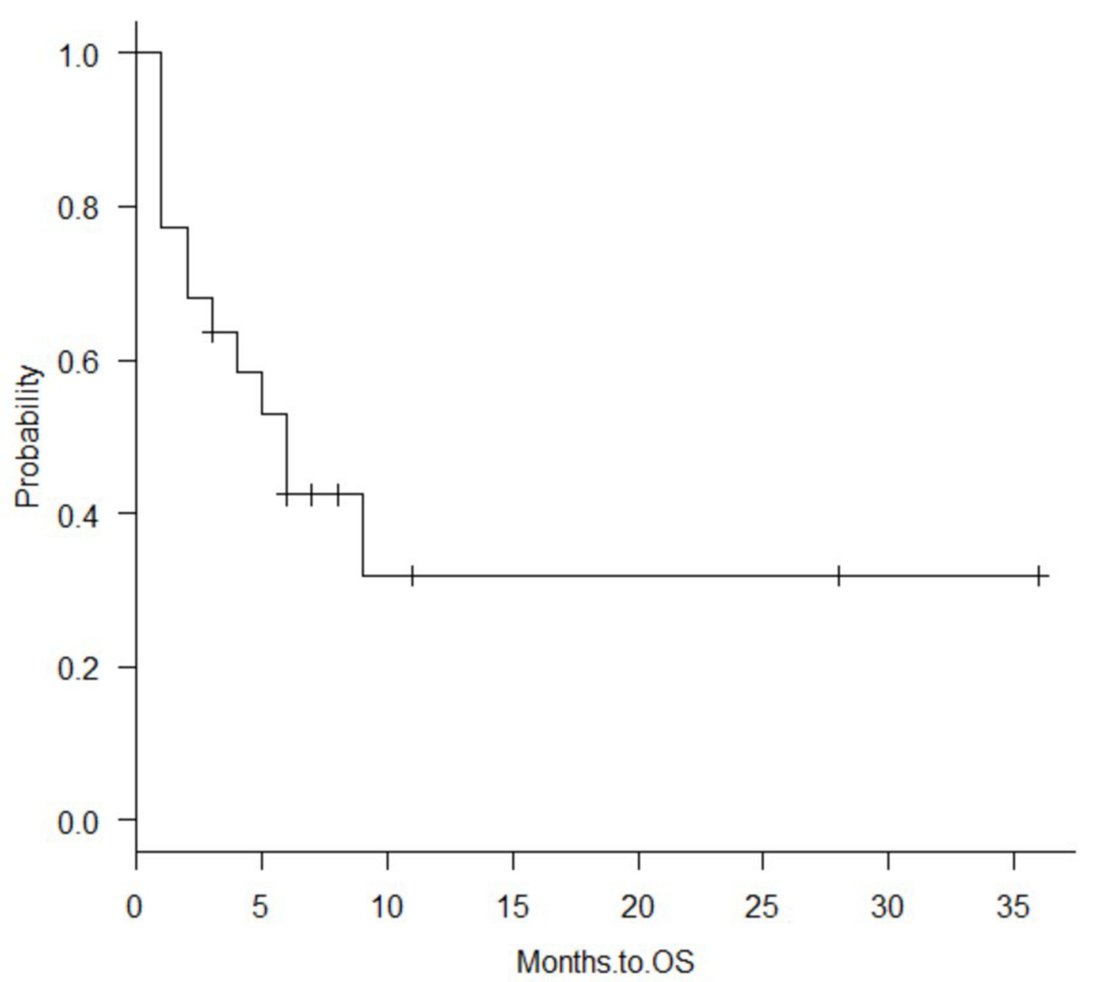

Figure I The one-year overall survival (OS) rate of 22 patients was $31.8 \%$, and the median survival time was 6 months (95\% Cl, $1-9$ months).

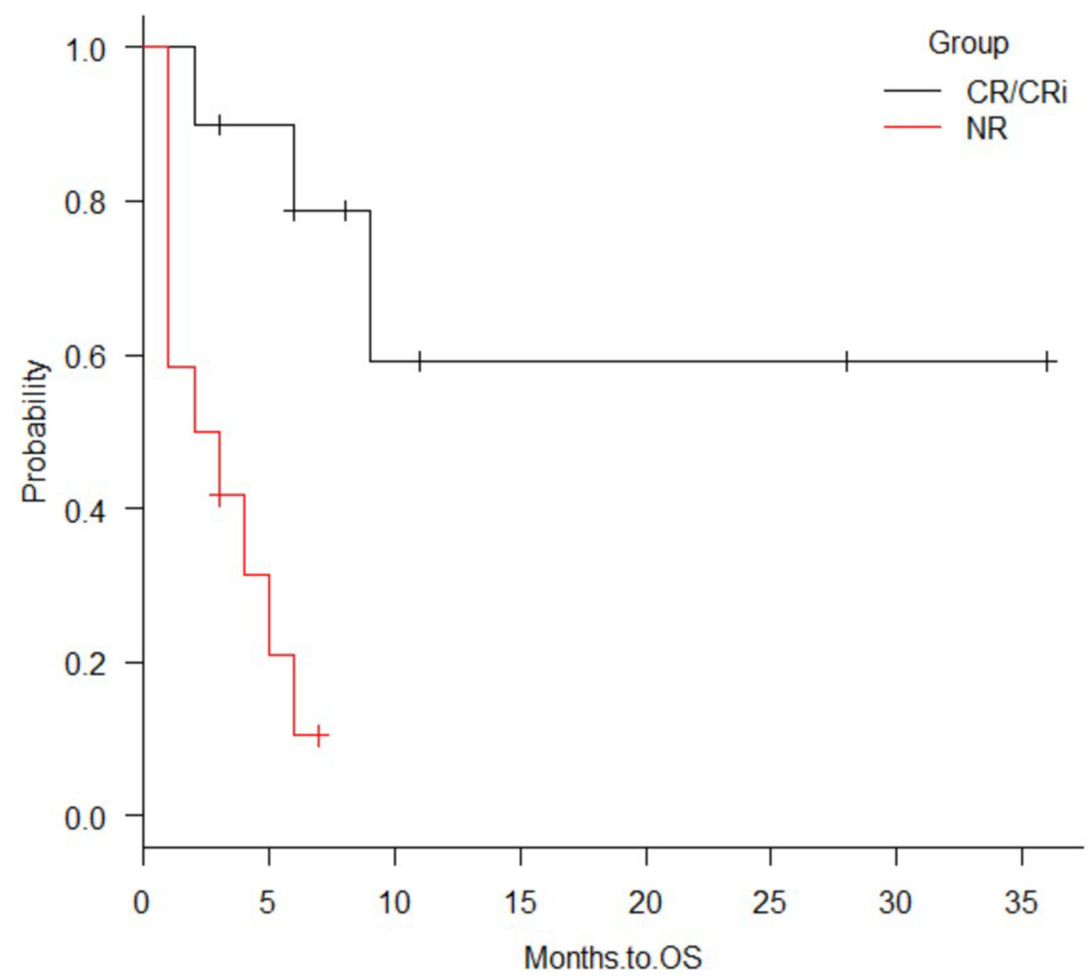

Figure 2 The one-year overall survival rate of 10 CR/CRi patients was $59.1 \%$, and the $12 \mathrm{NR}$ patients was $10.4 \%$, and there were statistical differences between the two groups $(\mathrm{P}=0.00 \mathrm{I})$. 
was $59.1 \%$, and the $12 \mathrm{NR}$ patients was $10.4 \%(\mathrm{p}=0.001)$ (Figure 2).

\section{Characteristics of the Patients Achieving Remission}

Among the $10 \mathrm{CR} / \mathrm{CRi}$ patients, four had positive FLT3ITD mutation and all of them received sorafenib treatment, one had FLT3-TKD, one had AML-ETO, one had CBF $\beta-$ MYH11 and two had NPM1 mutation.

Among the four FLT3-ITD patients, one was a patient relapsed after UCBT and remains disease-free currently for 28 months after venetoclax plus decitabine treatment; Two patients underwent UCBT after CR and remains disease-free for 8 and 11 months after complete remission; One patient relapsed 24 months after venetoclax treatment, but the blast counts remained below $10 \%$, and the patient is still alive.

Based on the treatment of venetoclax plus decitabine, one patient with FLT3-TKD obtained CRi after one course of the treatment and received HSCT; one AML-ETO positive relapsed AML patient with previous two times of transplant achieved $\mathrm{CR}$, however, the patient relapsed again after 7 months and died 9 months after CR; one patient with $\mathrm{CBF} \beta-\mathrm{MYH} 11$ acquired $\mathrm{CR}$ after one course of treatment, and now has a disease-free survival(DFS) of 6 months; one patient with hyperleukocytosis and a NPM1 gene mutation relapsed after 1 month and died 2 months after CR; one patient with a NPM1 gene mutation and normal white blood cell count acquired $\mathrm{CR}$ after one course of treatment, and now has a DFS of 3 months; one patient with no gene alterations relapsed after 4 months and died 6 months after CR (Figure 3).

All CR patients were de novo AML and no secondary AML. Six patients of ten had previously used hypomethylating agents before treatment $(60.0 \%)$. The median number of chemotherapies before treatment was $3(2-10)$ of the 10 patients.

\section{Characteristics of Patients Not Achieving Remission}

Among the 12 patients, two had FLT3-ITD mutations and both of them received sorafenib treatment, one patient had MLL-AF6, one case with hyperleukocytosis and NPM1 gene mutation, one had KRAS and ASXL1 mutation at the same time, one had NPM1 and TET-2 mutation, one had AML-ETO and IDH1 mutation. Four secondary AML were all refractory and did not get CR after the treatment of venetoclax plus decitabine. Eleven patients had previously used the hypomethylating agents before treatment (91.7\%). The median number of chemotherapies before treatment was $6(3-7)$ of the 12 patients.

\section{Differences in Characteristics Between CR/CRi and NR Patients}

The incidences of FLT3 mutation and NPM1 mutation were slightly higher in the $\mathrm{CR} / \mathrm{CRi}$ group $(50.0 \%, 50.0 \%)$ than in the

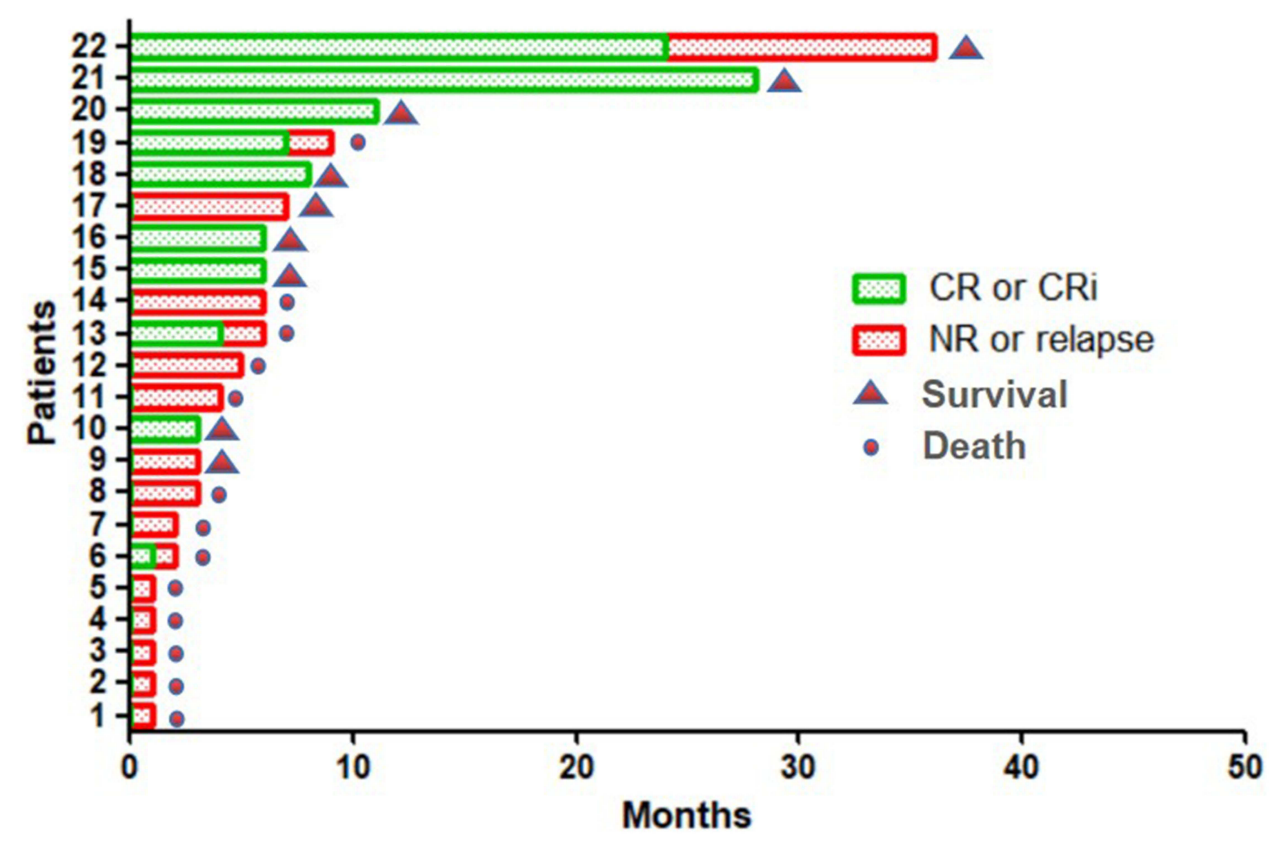

Figure 3 Remission and survival of the 22 patients. 
NR group (16.7\%, 16.7\%), but there were no significant differences $(p=0.17,0.17)$. The number of previous chemotherapies in the $\mathrm{CR} / \mathrm{CR}$ group was less than that in the NR group [3 (2-10) vs 6(3-7), $\mathrm{p}=0.05]$. All Secondary AML did not receive $\mathrm{CR} / \mathrm{CRi}$, and the incidence of secondary AML was slightly higher in the NR group (33.0\%) than in the CR/CRi group $(0 \%)(p=0.09)$. The proportion of patients using hypomethylating agents in the previous treatment in the CR/CRi group was lower than that in the NR group, but with no statistical difference between the two groups $(60.0 \%$ vs $91.7 \%, \mathrm{p}=0.21)$ (Table 1).

\section{Adverse Events}

No tumour lysis syndrome (TLS) was found in patients treated with increasing doses of venetoclax. Nausea and vomiting occurred in 11 patients $(50.0 \%)$. All patients had grade IV neutropenia and thrombocytopenia $(100 \%)$. No damage to liver and kidney function was observed. Seven patients temporarily discontinued treatment because they developed severe neutropenia (neutrophils $<0.1 \times 10^{9} / \mathrm{L}$ ). Pneumonia occurred in 14 patients $(63.6 \%)$, and septicaemia occurred in 2 patients $(9.0 \%)$. The cause of death in all patients was primary disease progression, and no patients died due to the side effects.

\section{Discussion}

Venetoclax has been widely used in the treatment of a variety of haematological tumours because of its killing effects on a variety of tumour cells. ${ }^{6}$ In AML, venetoclax can selectively kill AML cells and increase the sensitivity of AML cells to chemotherapy. ${ }^{7}$ However, a Phase II clinical study by Konopleva et $\mathrm{al}^{8}$ found that the efficacy of venetoclax alone in the treatment of AML was not ideal. In January 2018, a phase IB clinical trial published in Lancet Oncol combined venetoclax with hypomethylating agents in the treatment of elderly patients with AML, and the results showed that the effectiveness was significantly higher than that seen with venetoclax alone, ${ }^{9}$ and a recent study with more patients obtained the similar results. ${ }^{10}$

Given the results of these clinical trials, in November 2018, the US FDA approved the use of venetoclax combined with hypomethylating agents in the treatment of newly diagnosed AML patients who were not suitable for intensive chemotherapy. However, AML has a high disease recurrence rate, and some newly diagnosed AML are primary refractory AML.
Therefore, venetoclax combined with methylating agents has been used in the treatment of RR-AML, and has obtained good clinical results. Bewersdorf et al $^{11}$ published a meta-analysis on the treatment of $\mathrm{R} /$ R-AML with venetoclax alone or in combination with hypomethylating agents, and analyzed a total of 7 clinical studies $(n=219)$. For all studies combined, ORR was $31.1 \%$ including $20.7 \%$ for venetoclax monotherapy and $38.7 \%$ for Venetoclax combined with HMA/ LDAC.

We reviewed twelve published literatures from 2017 to 2020 on the treatment of R/R AML with venetoclax combined with hypomethylating agents..$^{3,4,12-21}$ The reported

Table 3 Predictive Gene Mutation in the Literatures

\begin{tabular}{|c|c|c|c|}
\hline & Favourable & No Impact & Adverse \\
\hline RUNXI & $\begin{array}{l}\text { DiNardo } C D,^{3} \text { Yu- } \\
\text { Wen Wang }{ }^{17}\end{array}$ & Erika Morsia $^{19}$ & \\
\hline IDHI/2. & $\begin{array}{l}\text { DiNardo } C D,^{3} \\
\text { DiNardo } C D,{ }^{16} \\
\text { Yinjun Lou }^{18}\end{array}$ & Erika Morsia $^{19}$ & \\
\hline FLT3-ITD & DiNardo CD. ${ }^{16}$ & $\begin{array}{l}\text { Aldoss I, } \\
\text { Yinjun Lou, }^{18} \\
\text { Erika Morsia }^{19}\end{array}$ & $\begin{array}{l}\text { Goldberg } \\
\text { AD, }{ }^{12} \text { Yu- } \\
\text { Wen Wang, }{ }^{17}\end{array}$ \\
\hline TP53 & & $\begin{array}{l}\text { Aldoss I, } \\
\text { Yinjun Lou, }^{18} \\
\text { Erika Morsia }^{19}\end{array}$ & $\begin{array}{l}\text { DiNardo } \\
C D{ }^{16}{ }^{1} \text { - } \\
\text { Wen Wang }^{17}\end{array}$ \\
\hline NPMI & $\begin{array}{l}\text { DiNardo CD, }{ }^{16} \\
\text { Yu-Wen Wang }\end{array}$ & Yinjun Lou ${ }^{18}$ & \\
\hline RAS & & & $\begin{array}{l}\text { Goldberg } \\
\text { AD, }{ }^{12} \text { Yu- } \\
\text { Wen Wang }\end{array}$ \\
\hline ASXLI & $\begin{array}{l}\text { Aldoss } \mathrm{I}^{15}{ }^{15} \text { Yu- } \\
\text { Wen Wang }\end{array}$ & & \\
\hline TET2 & Aldoss $1^{15}$ & & \\
\hline U2AFI & & & Aldoss ${ }^{1 .}{ }^{15}$ \\
\hline SRSF2 & Yu-Wen Wang ${ }^{17}$ & & \\
\hline SETBPI & & & $\begin{array}{l}\text { Yu-Wen } \\
\text { Wang }\end{array}$ \\
\hline DNMT3A & Erika Morsia $^{19}$ & & $\begin{array}{l}\text { Yu-Wen } \\
\text { Wang }^{17}\end{array}$ \\
\hline JAK2 & Erika Morsia ${ }^{19}$ & & \\
\hline BCOR & Erika Morsia ${ }^{19}$ & & \\
\hline
\end{tabular}


ORR rate was 44.5 (21-75) \%, CR/CRi was 39.8 (12-51)

$\%$. Compared with previous data, our study obtained the similar results, of which the ORR was $45.5 \%$ and the CR rate was $40.9 \%$, although these RR-AML patients were heavily pre-treated. Some clinical studies analyzed the effect of genes mutations on disease remission, and they concluded that the most favourable gene mutations are IDH1/2, NPM1 and ASXL1 genes, the unfavourable gene is RAS gene, and the most controversial genes are FLT3-ITD and TP53 mutations (Table 3). Venetoclax combined with FLT3 inhibitors can significantly improve the efficacy compared to the FLT3 inhibitors alone. Raghuveer Singh Mali et $\mathrm{al}^{22}$ reported that in vivo, venetoclax combined with quizartinib, a potent FLT3 inhibitor, showed greater anti-tumor efficacy compared to quizartinib or venetoclax alone. In our study, 5/7 FLT3-positive patients achieved CR/CRi. Of these, 4 patients were treated with the FLT3-ITD inhibitor sorafenib. Therefore, in our study, the high CR rate of FLT3-positive patients may be due to the use of FLT3 inhibitors, or may be venetoclax can overcome the poor prognosis of FLT3 mutation.

Although venetoclax can achieve good therapeutic effects, drug resistance often occurs. Patients who have previously received hypomethylating agents will develop resistance to treatment based on venetoclax, and the underlying mechanism needs to be further explored. Long-term efficacy is difficult to maintain for venetoclax. In a study of 43 relapsed or refractory patients treated with venetoclax in combination with HMAs, LDAC, or other agents such as cladribine or midostaurin, the ORR was $21 \%{ }^{3}$ Finally, although the majority of newly diagnosed patients respond to venetoclax-based combinations, the median duration of response may only be approximately 12 months, which necessitates an understanding of how resistance evolves and how this can be targeted. ${ }^{23}$ In our study, we also found that four of $10 \mathrm{CR} / \mathrm{CRi}$ patients relapsed, and the median time to relapse was only 5 (1.0-24) months. Therefore, haematopoietic stem cell transplantation (HSCT) is recommended for consolidation therapy in patients with CR after Venetoclax-based treatment; however, most of these patients are ineligible for HSCT due to their old age or poor performance status.

One of the major obstacles limiting the clinical use of venetoclax is tumour lysis syndrome (TLS). Currently, strategies slowly increasing the dose of venetoclax are used to reduce the risk of TLS. The most common adverse reaction is gastrointestinal symptoms. Due to the fact that high-dose venetoclax (1200mg daily) can cause serious gastrointestinal symptoms,${ }^{24}$ a dose of $400-800 \mathrm{mg} /$ day is widely used when venetoclax is combined with other drugs. Other serious adverse reactions include pneumonia, febrile neutropenia, or autoimmune haemolytic anaemia. ${ }^{25}$ In our study, there were no cases of treatment interruption or death due to the adverse drug reactions. The limited number of cases and the short follow-up time of some patients are the limitations of our study. In the future, we will continue to expand the number of cases, analyze more different gene mutations, try to find out which patients can benefit from this treatment, and strive to draw more reliable conclusions.

\section{Conclusions}

Venetoclax combined with a hypomethylating agent decitabine- based treatment can result in CR as well as complete molecular remission for some heavily pre-treated RR-AML patients. The treatment was well tolerated, and no serious adverse reactions occurred in this study of a real-world setting. Despite the success of the combination of venetoclax with a hypomethylating agent in inducing remission in RR-AML, acquisition of resistance to venetoclax in RR-AML is still the main cause of treatment failure. Therefore, HSCT is recommended for consolidation therapy in patients who achieve CR.

\section{Acknowledgments}

This work was partly supported by Key research and development projects in Anhui Province (NO.202004j07020019 and 202004j07020025), the Fundamental Research Funds for the Central Universities of China (NO. WK9110000109 and WK9110000003).

\section{Author Contributions}

JT collected data and wrote the original paper and analyzed data. CZ designed research, performed research, analyzed and interpreted data, and critically reviewed the manuscript. All authors made substantial contributions to conception and design, acquisition of data, or analysis and interpretation of data; took part in drafting the article or revising it critically for important intellectual content; agreed to submit to the current journal; gave final approval of the version to be published; and agree to be accountable for all aspects of the work.

\section{Disclosure}

The authors declare that they have no conflicts of interest. 


\section{References}

1. Perl AE, Altman JK, Cortes J, et al. Selective inhibition of FLT3 by gilteritinib in relapsed or refractory acute myeloid leukaemia: a multicentre, first-in-human, open-label, Phase 1-2 study. Lancet Oncol. 2017;18(8):1061-1075. doi:10.1016/S1470-2045(17)30416-3

2. Burnett AK, Hills RK, Nielsen OJ, et al. A comparison of FLAG-Ida and daunorubicin combined with clofarabine in high-risk acute myeloid leukaemia: data from the UK NCRI AML17 Trial. Leukemia. 2018;32(12):2693-2697. doi:10.1038/s41375-018-0148-3

3. DiNardo CD, Rausch CR, Benton C, et al. Clinical experience with the BCL2-inhibitor venetoclax in combination therapy for relapsed and refractory acute myeloid leukemia and related myeloid malignancies. Am J Hematol. 2018;93(3):401-407. doi:10.1002/ ajh. 25000

4. Shahswar R, Hamwi I, Lueck C, et al. Registry for the off-label use of venetoclax in patients with relapsed or refractory acute myeloid leukemia. HemaSphere. 2018;2(Supplement 2):794.

5. Hallek M, Cheson BD, Catovsky D, et al. Diagnosis and management of AML in adults: 2017 ELN recommendations from an international expert panel. Blood. 2017;129(4):424-447.

6. Roberts AW, Davids MS, Pagel JM, et al. Targeting BCL2 with Venetoclax in Relapsed Chronic Lymphocytic Leukemia. $N$ Engl $J$ Med. 2016;374(4):311-322. doi:10.1056/NEJMoa1513257

7. Chan SM, Thomas D, Corces-Zimmerman MR, et al. Isocitrate dehydrogenase 1 and 2 mutations induce BCL-2 dependence in acute myeloid leukemia. Nat Med. 2015;21(2):178-184. doi:10.1038/nm.3788

8. Konopleva M, Pollyea DA, Potluri J, et al. Efficacy and biological correlates of response in a phase II study of venetoclax monotherapy in patients with acute myelogenous leukemia [J]. Cancer Discov. 2016;6(10):1106-1117. doi:10.1158/2159-8290.CD-16-0313

9. DiNardo CD, Pratz KW, Letai A, et al. Safety and preliminary efficacy of venetoclax with decitabine or azacitidine in elderly patients with previously untreated acute myeloid leukaemia: a non-randomised, open-label, phase 1b study. Lancet Oncol. 2018;19 (2):216-228. doi:10.1016/S1470-2045(18)30010-X

10. DiNardo CD, Pratz K, Pullarkat V, et al. Venetoclax combined with decitabine or azacitidine in treatment-naive, elderly patients with acute myeloid leukemia. Blood. 2019;133(1):7-17. doi:10.1182/ blood-2018-08-868752

11. Bewersdorf JP, Giri S, Wang R. Venetoclax as monotherapy and in combination with hypomethylating agents or low dose cytarabine in relapsed and treatment refractory acute myeloid leukemia: a systematic review and meta-analysis. Haematologica. 2020;105 (11):2659-2663. doi:10.3324/haematol.2019.242826

12. Goldberg AD, Horvat TZ, Hsu M, et al. Venetoclax combined with either a hypomethylating agent or low-dose cytarabine shows activity in relapsed and refractory myeloid malignancies. Blood. 2017;130 (Supplement 1):1353.
13. Aldoss I, Yang D, Aribi A, et al. Efficacy of the combination of venetoclax and hypomethylating agents in relapsed/refractory acute myeloid leukemia. Haematologica. 2018;103(9):e404-e407. doi:10.3324/haematol.2018.188094

14. Ram R, Amit O, Zuckerman T, et al. Venetoclax in patients with acute myeloid leukemia refractory to hypomethylating agents-a multicenter historical prospective study. Ann Hematol. 2019;98(8):1927-1932. doi:10.1007/s00277-019-03719-6

15. Aldoss I, Yang D, Pillai R, et al. Association of leukemia genetics with response to venetoclax and hypomethylating agents in relapsed/ refractory acute myeloid leukemia. Am J Hematol. 2019;94(10): E253-E255. doi:10.1002/ajh.25567

16. DiNardo CD, Maiti A, Rausch CR, et al. 10-day decitabine with venetoclax for newly diagnosed intensive chemotherapy ineligible, and relapsed or refractory acute myeloid leukaemia: a single-centre, Phase 2 trial. Lancet Haematol. 2020;7(10):e724-e736. doi:10.1016/ S2352-3026(20)30210-6

17. Wang Y-W, Tsai C-H, Lin C-C, et al. Cytogenetics and mutations could predict outcome in relapsed and refractory acute myeloid leukemia patients receiving BCL-2 inhibitor venetoclax. Ann Hematol. 2020;99(3):501-511. doi:10.1007/s00277-020-03911-z

18. Lou Y, Shao L, Mao L, et al. Efficacy and predictive factors of venetoclax combined with azacitidine as salvage therapy in advanced acute myeloid leukemia patients: a multicenter retrospective study. Leuk Res. 2020;91:106317. doi:10.1016/j.leukres.2020.106317

19. Morsia E, McCullough K, Joshi M, et al. Venetoclax and hypomethylating agents in acute myeloid leukemia: mayo Clinic series on 86 patients. Am J Hematol. 2020;95(12):1511-1521. doi:10.1002/ ajh. 25978

20. Gaut D, Burkenroad A, Duong T, et al. Venetoclax combination therapy in relapsed/refractory acute myeloid leukemia: a single institution experience. Leuk Res. 2020;90:106314. doi:10.1016/j. leukres.2020.106314

21. Ganzel C, Ram R, Gural A, et al. Venetoclax is safe and efficacious in relapsed/refractory AML. Leuk Lymphoma. 2020;61 (9):2221-2225. doi:10.1080/10428194.2020.1761964

22. Mali RS, Zhang Q, DeFilippis R, et al. Venetoclax combines synergistically with FLT3 inhibition to effectively target leukemic cells in FLT3-ITD+ acute myeloid leukemia models. Haematologica. 2020. haematol. 2019;244020.

23. Wilde L, Ramanathan S, Kasner M. B-cell lymphoma-2 inhibition and resistance in acute myeloid leukemia. World J Clin Oncol. 2020;11(8):528-540. doi:10.5306/wjco.v11.i8.528

24. Eyre TA, Roeker LE, Fox CP, et al. The efficacy and safety of venetoclax therapy in elderly patients with relapsed, refractory chronic lymphocytic leukaemia. $B r \quad J$ Haematol. 2020;188 (6):918-923. doi:10.1111/bjh.16271

25. DiNardo CD, Jonas BA, Pullarkat V, et al. Azacitidine and Venetoclax in Previously Untreated Acute Myeloid Leukemia. $N$ Engl J Med. 2020;383(7):617-629. doi:10.1056/NEJMoa2012971
Cancer Management and Research

\section{Publish your work in this journal}

Cancer Management and Research is an international, peer-reviewed open access journal focusing on cancer research and the optimal use of preventative and integrated treatment interventions to achieve improved outcomes, enhanced survival and quality of life for the cancer patient.
The manuscript management system is completely online and includes a very quick and fair peer-review system, which is all easy to use. Visit http://www.dovepress.com/testimonials.php to read real quotes from published authors. 